\title{
El uso de la evidencia en las políticas públicas: el caso de las Evaluaciones de Desempeño y Ejecución Presupuestal en el sector Educación en el Perú
}

\author{
The use of evidence in public policies: the case of Performance \\ Evaluations and Budgetary Execution in the Education sector in Peru
}

\author{
Alejandra Navarro Véliz (*) \\ Enacción Consultores \\ ORCID: 0000-0002-8135-098X
}

Antonio Zuñiga Romero (**)

Pontificia Universidad Católica del Perú

ORCID: 0000-0002-0206-3107

Lina Arenas Romero (***)

Pontificia Universidad Católica del Perú

ORCID: 0000-0002-4341-6343

Fecha de recepción: 27 de agosto

Fecha de aceptación: 16 de noviembre

ISSN en línea: $2415-2498$

Navarro, A.; A. Zuñiga y L. Arenas (2018) «El uso de la evidencia en las políticas: el caso de las Evaluaciones de Desempeño y Ejecución Presupuestal en el sector Educación en el Perú». Politai: Revista de Ciencia Política, Año 9, segundo semestre № 17: pp. 119-146.

DOI: https://doi.org/10.18800/politai.201802.004

* Licenciada en Ciencia Política Y Gobierno (PUCP), consultora de Enacción. navarro.alejandra@pucp.edu.pe

** Licenciado en Ciencia Política y Gobierno (PUCP), pre docente en la PUCP. a20083191@pucp.pe

***Licenciada en Psicología Social (PUCP), Master en Desarrollo Social y Políticas Sociales (Manchester UK) y Docente en la PUCP. larenas@pucp.edu.pe 


\title{
Sumilla
}

El presente artículo pretende aproximarse al uso la evidencia en la formulación de políticas públicas. Para ello se analiza las evaluaciones independientes del sector Educación, en particularmente las Evaluaciones de Desempeño y Ejecución Presupuestal (EDEP), las que tomamos como estudio de caso. Con el fin de presentar el contexto en el que se ubica esta herramienta, en primer lugar se presenta un breve recuento de la literatura en torno al uso de la evidencia en las políticas públicas y a la relación entre el conocimiento y las políticas públicas en el Perú. En segundo lugar se presenta el análisis de cinco EDEP del sector Educación a la luz de los siguientes ejes de análisis: i) el perfil de los investigadores y la metodología empleada en la investigación, y ii) el análisis del cumplimiento de los compromisos consignados. El artículo concluye que las EDEP generan un grado intermedio en el cumplimiento de sus recomendaciones, lo que esta mediado, entre otros factores, por el tipo de intervención pública evaluada; y cuestiona la utilidad de las EDEP como mecanismo efectivo de evaluación y mejora del diseño o componente de un programa, identificando oportunidades de mejora de la herramienta, como el rediseño metodológico, la simplificación en el reporte de resultados, la potenciación de la matriz y sus mecanismos, entre otros.

Palabras clave: Uso de evidencia, formulación de políticas públicas, Evaluaciones de desempeño y Ejecución Presupuestal

\begin{abstract}
This article aims to approach the use of evidence in the formulation of public policies. To this end, we analyze the independent evaluations of the Education sector, particularly the Performance Evaluations and Budget Execution (EDEP - for its acronym in Spanish), which we take as a case study. In order to present the context in which this tool is located, first a brief account of the literature is presented about the use of evidence in public policies and the relationship between knowledge and public policies in Peru. . Secondly, the analysis of five EDEPs of the Education sector is presented in the light of the following axes of analysis: i) the profile of the researchers and the methodology used in the research, and ii) the analysis of the fulfillment of the consigned commitments. The article concludes that EDEPs generate an intermediate degree in compliance with their recommendations, which is mediated, among other factors, by the type of public intervention evaluated; and questions the usefulness of the EDEP as an effective mechanism for evaluating and improving the design or component of a program, identifying opportunities to improve the tool, such as the methodological redesign, the simplification in the reporting of results, the empowerment of the matrix and its mechanisms, among others.
\end{abstract}

Keywords: Use of evidence, formulation of public policies, Performance Evaluations and Budget Execution 


\section{Introducción: Presupuesto informado por evidencia}

El proceso de modernización del Estado Peruano trajo consigo la tecnocratización del sector público, especialmente en sectores vinculados a la economía (Dargent, 2008), y que posteriormente se fue consolidando en sectores que atienden la política social (Vela et al., 2014). Es en este marco de alto nivel de especialización técnica de ciertas carteras del sector público, que desde el año 2008 se opta por implementar de manera progresiva el Presupuesto por Resultados como una de las principales herramientas de gestión pública. Este tiene como objetivo vincular la asignación de recursos a productos y resultados medibles a favor de la población. Esta transformación en el Sistema Nacional de Presupuesto Público (SNPP) supuso una transición entre una asignación inercial, o presupuesto histórico, hacia la gestión orientada a resultados a través de un presupuesto informado. Esta etapa de la historia del presupuesto nacional tiene ya diez años de ser implementada, y cuenta como dispositivo principal a la Ley General $\mathrm{N}^{\circ} 28411$, recientemente modificada a través del $\mathrm{DL} \mathrm{N}^{\circ} 1440$, lo que marca una nueva etapa en la conceptualización no sólo del SNPP, sino también del uso de la evidencia para informar el diseño, ejecución y evaluación de las políticas públicas nacionales, materia de discusión en el presente artículo.

El Presupuesto por Resultados cuenta con diversos instrumentos para su implementación. Uno de ellos son los Programas Presupuestales, los que a través del desarrollo metodológico en su diseño permiten agrupar productos y actividades que contribuyen a logros específicos que atienden objetivos de política nacional. Los otros instrumentos refieren al seguimiento, las evaluaciones, y los incentivos presupuestarios. Si bien todos los sectores utilizan estos instrumentos en las diferentes fases del proceso presupuestario, el sector Educación ha sido uno de los primeros partícipes de esta transformación, al ser uno de los primeros en programar gran parte de sus recursos en los diversos programas diseñados para atender sus principales problemáticas. Son sus ocho programas presupuestales los que desde el año 2008 concentran la mayor parte de los recursos dirigidos a la provisión del servicio educativo. Es así que desde el sector se ha venido promoviendo una agenda de generación y uso de evidencia desde aquel entonces. El sector cuenta en la actualidad con una serie de evaluaciones independientes, herramientas para el monitoreo, e incluso desde hace algunos años con un laboratorio de innovación que informa a las políticas desde la evidencia, MineduLab.

Dentro de los diversos tipos de evaluación que el sector ha desarrollado, o de los que se ha visto sujeto, se encuentran las Evaluaciones de Desempeño y Ejecución Presupuestal (EDEP), que son un tipo de evaluación independiente que pretende informar a la asignación presupuestal de diferentes intervenciones, que pueden constituir o no un Programa Presupuestal. Estas evaluaciones, luego de ser ejecutadas, cierran el ciclo con la firma de una matriz de compromisos entre los principales sectores responsables del cumplimiento. Estos compromisos son producto de los hallazgos críticos de cada evaluación. El sector Educación firma su primera matriz de compromisos el año 2012, dirigida a las acciones de mantenimiento de la infraestructura educativa. En los años sucesivos se firmaron matrices para otras intervenciones del servicio educativo, como por ejemplo las de Acompañamiento Pedagógico, Materiales Educativos, PRONIED y Beca 18, entre otras. Dichas evaluaciones y sus resultados son materia de la presente investigación. 
El presente artículo pretende aproximarse al uso la evidencia en la formulación de políticas públicas. Con este propósito utilizamos como proxy a las evaluaciones independientes del sector Educación, particularmente a las Evaluaciones de Desempeño y Ejecución Presupuestal (EDEP), las que tomamos como estudio de caso, ya que estas informan a través de la evidencia del desempeño de la intervención pública. Con el fin de presentar el contexto en el que se ubica esta herramienta, en primer lugar se presenta un breve recuento de la literatura en torno al uso de la evidencia en las políticas públicas y a la relación entre el conocimiento y las políticas públicas en el Perú. En segundo lugar se presenta el análisis de cinco EDEP del sector Educación a la luz de los siguientes ejes de análisis: i) el perfil de los investigadores y la metodología empleada en la investigación, y iii) el análisis del cumplimiento de las compromisos consignados. Finalmente se esbozan conclusiones y se trazan reflexiones con el propósito de orientar la agenda y siguientes pasos en la investigación acerca del uso de la evidencia en las políticas públicas.

\section{Marco teórico: Políticas públicas basadas en evidencia y la relación entre el conocimiento y las políticas públicas}

Existe actualmente un consenso mundial acerca de la importancia de elaborar políticas públicas utilizando evidencia. Los principales actores, los decisores de políticas y el sector de producción del conocimiento, no tardaron en ponerse de acuerdo en la relevancia. La información de la academia e investigación viene alimentando de manera sistemática y rigurosa el diseño de políticas en las últimas cinco décadas, inicialmente desde la ciencias de la salud (Oliver et al., 2014). Sin embargo la discusión de cómo las ciencias deben orientar el quehacer del Estado tiene larga data y está enraizada desde la creación de las mismas, particularmente de las ciencias sociales. En la actualidad, la discusión sobre las políticas públicas basadas en evidencia (EBP, evidence-based policy, por sus siglas en inglés) está más orientada a debatir en torno a la efectividad en el uso y en la implementación de las mismas, al proceso de puesta en práctica, y a sus persistentes limitaciones y retos.

Por ejemplo, la relación entre las ciencias sociales y las políticas sociales se documenta desde el desarrollo institucional de las primeras. Esta relación se sintetiza en dos corrientes opuestas que parten del supuesto que la promoción de políticas sociales no es en su origen políticamente neutral. Por ende, sería deber de los científicos sociales el asumir el rol neutral y estrictamente profesional para informar, a través de la recolección y análisis de data, al Estado. Las posiciones encontradas nacen de este supuesto. Mientras que la neutralidad axiológica de esta tradición representada por Weber se acerca más al deber ser empiricista que toma la ciencia como verdad, la corriente que tiene a Gramsci como uno de sus principales actores, postula al pensamiento crítico versus la neutralidad, como uno de sus principios (Lee et al., 2005). Los escenarios que trascienden al debate sobre el uso de evidencia, discuten si para la generación de políticas es acaso solamente necesario el conocimiento e investigación, o si las políticas no deberían girar exclusivamente en torno a la evidencia; e introducen variables como movilización social, creatividad social, soporte político y en general, a la política de las políticas como variables también ineludibles en la generación o rechazo de propuestas de políticas públicas (Milani, 2005; O’Dwyer, 2004).

Asimismo, se debe precisar que la formulación de políticas públicas hace uso de diversas fuentes. El uso de la evidencia científica para informar la toma de decisiones es sólo una de ellas; teóricamente, la de mayor peso y legitimidad según los axiomas de neutralidad arriba descritos. Sin embargo, y como hemos visto, la orientación y diseño de las políticas públicas puede conducirse 
también en torno a decisiones de agenda gubernamental de los grupos de poder y prioridades nacionales de atención, entre otros. Justamente uno de los propósitos principales de diseñar políticas basadas en evidencia es hacerlas resistentes y blindarlas a presiones políticas y a grupos de intereses externos que no estén alineados con los objetivos de política nacionales. Desde esta perspectiva se entiende a la ciencia como un contrapeso para reducir el efecto de intereses políticos (Leuz, 2018; Zingales 2015). Es por esto que las EBP cumplen un rol fundamental en el fortalecimiento de la democracia y sus pilares (como por ejemplo la rendición de cuentas), y por ende forman parte de un componente importante en la agenda de organismos multilaterales y cooperantes que buscan promover la democracia en el mundo, así como financiar, respaldar y publicar investigaciones en torno a la efectividad de políticas que usen evidencia (Milani, 2005; Prewiott, 2012).

En esa línea, un interesante estudio de Garretón, Cruz y Espinoza (2010) analiza las relaciones entre las ciencias sociales y la formulación y gestión de las políticas públicas en Chile, centrándose en cuatro aspectos: i) quiénes y en qué condiciones investigan para el Estado, ii) el contenido de las investigaciones, iii) las perspectivas metodológicas, y finalmente, iv) los usos de las investigaciones. Dentro de las varias conclusiones a las que llega, resaltan particularmente tres. La primera refiere a la debilidad de las investigaciones de dar cuenta de sus enfoques teóricos y metodológicos, así como de los pocos recursos humanos que estas involucran. La segunda se concentra en la multidisciplinariedad que requieren las investigaciones, debido a la movilización y manejo de distintas perspectivas y metodologías necesarias. Finalmente los temas analizados emergen de lo ya naturalizado como problemas sociales, dejando de lado espacio para temas de mejora de la gestión de políticas públicas.

En el estudio de las EBP han surgido diversas tipologías. Una de las más comprensivas y utilizadas postula cuatro principales categorías en torno a los usos que se le dan a las políticas basadas en evidencia: i) usos instrumentales, ii) usos conceptuales, iii) usos tácticos, y iv) usos impuestos (Weiss, 1979). Los usos instrumentales ocurren cuando el conocimiento es directamente aplicado a la toma de decisiones para abordar un problema en particular. Los usos conceptuales refieren a cuando la investigación informa sobre cómo los decisores de políticas deben pensar sobre los potenciales problemas o soluciones. Los usos tácticos involucran acciones estratégicas y simbólicas para apoyar o interpelar una idea, propuesta o programa específico, como una propuesta legislativa o un esfuerzo de reforma. Los usos impuestos, que se entienden como una variación de los instrumentales, describen disposiciones para aplicar el conocimiento, como que el presupuesto sea asignado en función a evidencia, o que las agencias desarrollen programas basados en evidencia. La literatura agrega una quinta categoría de uso simbólico, donde se genera o colecta evidencia con ninguna intención real de usarla, sino de persuadir sobre una determinada posición o retrasar la toma de decisiones de manera deliberada (Prewitt, 2012). De hecho, una de las quejas más frecuentes de la comunidad científica es que los decisores de políticas usan frecuentemente la evidencia solo para confirmar creencias pre establecidas. El contra argumento de los decisores es que una vez puesta la evidencia sobre la mesa, es difícil ignorarla, y ello abre espacios a debates de mejor calidad.

Es en esta misma línea que se genera uno de los principales retos de la investigación en EBP, que es conciliar a las posturas de las dos comunidades que la comprenden: los científicos sociales y los decisores de política. Esta perspectiva que explica la dificultad de llegar a acuerdos se le conoce como "la metáfora de las dos comunidades", que básicamente explica que ambos actores están separados por lenguajes y códigos diferentes, valores, normas, afiliaciones sociales y personales, hábitos de expresión, modos de pensamiento (deductivo versus inductivo), entre otras características. Mientras que los científicos tienen como meta la búsqueda sistemática para entender el mundo de una manera precisa y confiable, los decisores buscan respuestas prácticas a 
problemáticas particulares (Henig, 2009).

Finalmente, la investigación en EBP muestra adicionalmente una serie de limitaciones y retos a nivel macro y micro, sobre todo presentados en los meta análisis críticos que abordan el tema (Oliver et al., 2014). A nivel micro, los hallazgos apuntan, por ejemplo, a la importancia de las relaciones personales y contacto entre decisores de política e investigadores, a la situación profesional de los investigadores y a cómo sus líneas de investigación e intereses se encuentran relacionados (Pawson and Tilley, 1996); mientras que los ejes centrales de debate en un nivel macro, parten de supuestos que cuestionan la priorización de cierto tipo de evidencia sobre otro tipo de información de menor rango o calidad; la ausencia de evaluaciones robustas que muestren que el uso de evidencia lleve a mejores resultados; y finalmente a que las reflexiones en torno a las EBP están por lo general más orientadas a encontrar cómo incrementar el impacto de las investigaciones, en lugar de entender el proceso mismo detrás de la elaboración de las políticas.

\section{La generación de conocimiento para informar las políticas públicas en el ámbito nacional}

De manera aplicada, la relación entre la generación de conocimiento y Estado puede tener, en buena cuenta, dos lecturas, o dos formas de ser entendida: una externa y otra interna. Ambas refieren al origen del conocimiento que informa al Estado, y se diferencian en tanto al actor que solicita la información o evidencia.

La lectura externa refiere al conocimiento producido por el interés o a solicitud de agentes externos al Estado. Es decir, refiere al conocimiento que viene de fuera de las agencias y entidades estatales, como por ejemplo desde los think tanks, las universidades, los centros de investigación, expertos o agencias multilaterales. Son estos actores externos quienes desarrollan las preguntas de investigación, seleccionan las intervenciones a ser evaluadas, desarrollan hipótesis, contratan personal que diseñe y ejecute la investigación, bajo los parámetros independientes que cada entidad se imponga. Por otro lado, la lectura interna refiere al conocimiento que informa al Estado, pero que tiene como principal actor que solicita la información o evidencia al mismo Estado. Se tratan de investigaciones, que si bien pueden denominarse independientes, son producidas por interés de alguna agencia estatal, bajo sus normas y parámetros de contratación para con quien las diseñe y ejecute. En este caso, las preguntas de investigación y aspectos de las intervenciones o programas a ser mirados con mayor relevancia son determinados por alguna agencia estatal, sea la rectora de los programas, o quien las financia. Es esta mirada a la producción de conocimiento solicitado desde el Estado, que denominamos lectura interna, la que resulta poco estudiada en el caso peruano. Sobre este tipo de conocimiento, existen brechas importantes de información: ¿en qué áreas o temáticas se han desarrollado mayores conocimientos?, ¿qué sectores carecen de investigación local?, ¿qué nivel de evidencia local existe?, ¿quiénes son los expertos de cada sector?, ¿qué perfil tienen?, ¿son realmente independientes?, ¿se produce conocimiento sistemático?, ¿desde cuándo?, ¿quiénes lo incentivan?, ¿qué entidades o agencias son las que más financian generación de conocimiento en el país?

De otro lado, la problemática descrita está también relacionada con las ciencias que están detrás de este conocimiento, las que inexorablemente imponen sus tradiciones, marcos teóricos y principales 
herramientas de análisis. En gran cuenta, la inserción de las ciencias sociales en el Estado, sobre todo la economía como disciplina predominante, juega un rol central en la producción del conocimiento. Debido a ello, resulta clave entender cómo los enfoques, preferencias metodológicas, y bases epistemológicas propias de esta disciplina han moldeado buena parte de la forma en la que se genera y se analiza el conocimiento desde el Estado. Sobre el particular surgen diversos cuestionamientos: ¿dimensiones del desempeño como eficacia y eficiencia aseguran necesariamente un mejor servicio al ciudadano? Asimismo, ¿cuál es el rol que debería tener la ciencia política en este proceso? Si se considera que incluso las arenas "técnicas" se ven influenciadas por la política, ¿hasta qué punto la autonomía ganada por ciertos sectores altamente especializados se debe a ese conocimiento experto que ha generado? ¿Si se generara el mismo capital humano en otros sectores del Estado, se produciría algún cambio en el balance de poder entre estas instituciones?, ¿qué buenas o malas decisiones se han tomado, y en qué sectores particularmente, utilizando a la evidencia como justificación o dándole un uso simbólico?

\section{Las Evaluaciones de Desempeño y Ejecución Presupuestal en el sector Educación}

En el transcurso de los últimos años se han elaborado un total de 11 EDEP sobre el sector Educación. Sin embargo, la presente investigación analiza cinco, ya que son las que se encuentran vigentes. A continuación se presenta cada una de las evaluaciones: se empieza describiendo el objetivo de la intervención pública evaluada y en qué consistió la evaluación, posteriormente se analiza a los evaluadores y la metodología que se emplearon en las EDEP. Finalmente, se presenta el análisis del cumplimiento de los compromisos.

\begin{tabular}{|c|c|c|}
\hline Intervención Pública & $\begin{array}{c}\text { Sumilla de la Intervención } \\
\text { Pública }\end{array}$ & Sumilla de la EDEP \\
\hline Materiales Educativos & $\begin{array}{l}\text { El fin de la política de materiales } \\
\text { es mejorar los niveles de } \\
\text { aprendizaje de los estudiantes en } \\
\text { las cuatro principales áreas } \\
\text { curriculares: Comunicación } \\
\text { Integral, Lógico Matemática, } \\
\text { Personal Social y Ciencia y } \\
\text { Ambiente. Para ello se tienen que } \\
\text { cumplir los propósitos de } \\
\text { dotación de materiales alienados } \\
\text { al currículo y pertinentes al } \\
\text { contexto, pero también se debe } \\
\text { cumplir con el dominio y uso de } \\
\text { los mismos de parte de los } \\
\text { docentes. }\end{array}$ & $\begin{array}{l}\text { Evaluación centrada en los } \\
\text { materiales impresos que } \\
\text { MINEDU distribuye a todas } \\
\text { las escuelas públicas } \\
\text { primarias de la Educación } \\
\text { Básica Regular; } \\
\text { específicamente, a los textos } \\
\text { integrados y libros para las } \\
\text { cuatro áreas mencionadas, de } \\
\text { primer a sexto grado. }\end{array}$ \\
\hline $\begin{array}{c}\text { Programa Nacional } \\
\text { Beca } 18\end{array}$ & $\begin{array}{l}\text { La intervención tiene por } \\
\text { objetivo, a nivel de resultado } \\
\text { final, lograr la inserción en el } \\
\text { mercado laboral de aquellos }\end{array}$ & $\begin{array}{l}\text { Análisis del Programa } \\
\text { Nacional de Becas (BECA } \\
\text { 18), a partir de la } \\
\text { identificación de los puntos }\end{array}$ \\
\hline
\end{tabular}




\begin{tabular}{|c|c|c|}
\hline & $\begin{array}{l}\text { jóvenes egresados del nivel de } \\
\text { educación superior bajo un } \\
\text { estándar de calidad }\end{array}$ & $\begin{array}{l}\text { críticos en su desarrollo y } \\
\text { sobre el cual se hacen } \\
\text { recomendaciones }\end{array}$ \\
\hline $\begin{array}{l}\text { Acompañamiento } \\
\text { pedagógico }\end{array}$ & $\begin{array}{l}\text { El Acompañamiento Pedagógico } \\
\text { es una estrategia que consiste en } \\
\text { ofrecer una asesoría planificada, } \\
\text { continua y pertinente a los } \\
\text { docentes acompañados para } \\
\text { mejorar su práctica pedagógica } \\
\text { en aula y de gestión, a través de } \\
\text { la retroalimentación y soporte } \\
\text { técnico, lo cual debería generar } \\
\text { resultados concretos en los } \\
\text { aprendizajes de los estudiantes. }\end{array}$ & $\begin{array}{l}\text { Evaluación de diseño y } \\
\text { ejecución de presupuesto } \\
\text { referida al Acompañamiento } \\
\text { Pedagógico que se realiza } \\
\text { desde el año } 2008 \text { en } \\
\text { Instituciones Educativas } \\
\text { públicas multiedad de II } \\
\text { Ciclo (Inicial) y multigrado } \\
\text { de Primaria de la Educación } \\
\text { Básica Regular (EBR), en } \\
\text { particular aquellas que } \\
\text { atienden a población bilingüe } \\
\text { con enfoque EIB. }\end{array}$ \\
\hline $\begin{array}{l}\text { Programa Nacional de } \\
\text { Mantenimiento de } \\
\text { locales escolares }\end{array}$ & $\begin{array}{l}\text { El Programa de Mantenimiento } \\
\text { de Locales Escolares (PME) es } \\
\text { un mecanismo de transferencia } \\
\text { directa de recursos financieros, } \\
\text { los cuales se encuentran dirigidos } \\
\text { exclusivamente al mantenimiento } \\
\text { preventivo básico y asignados a } \\
\text { la unidad más pequeña y } \\
\text { desconcentrada del aparato de } \\
\text { gestión de la educación en el } \\
\text { sector público: la institución } \\
\text { educativa. }\end{array}$ & $\begin{array}{l}\text { Análisis del Programa de } \\
\text { Mantenimiento de Locales } \\
\text { Escolares, a partir de la } \\
\text { identificación de puntos } \\
\text { críticos y los resultados de la } \\
\text { indagación a profundidad } \\
\text { sobre estos puntos críticos. }\end{array}$ \\
\hline $\begin{array}{l}\text { Programa Nacional de } \\
\text { Infraestructura } \\
\text { educativa }\end{array}$ & $\begin{array}{l}\text { El objetivo es contribuir con la } \\
\text { modernización educativa a nivel } \\
\text { nacional, brindando una } \\
\text { infraestructura educativa de } \\
\text { acuerdo con las normas y } \\
\text { lineamientos técnico- } \\
\text { pedagógicos establecidos por el } \\
\text { sector, en beneficio de la niñez y } \\
\text { juventud peruana. }\end{array}$ & $\begin{array}{l}\text { Evaluación del diseño de las } \\
\text { acciones vinculadas con el } \\
\text { PRONIED, a fin de } \\
\text { determinar el más apropiado } \\
\text { para la obtención de } \\
\text { resultados, a partir de la } \\
\text { identificación de los } \\
\text { productos que se financian } \\
\text { con la intervención y su } \\
\text { relación con los resultados } \\
\text { esperados. }\end{array}$ \\
\hline
\end{tabular}

Cuadro 1. Evaluaciones de Desempeño y Ejecución Presupuestal en el sector Educación. Elaboración propia. 


\section{¿Quiénes elaboraron las EDEP?}

\begin{tabular}{|c|c|c|}
\hline EDEP & $\begin{array}{c}\text { Año de } \\
\text { evaluación }\end{array}$ & Consultor \\
\hline \multirow{3}{*}{$\begin{array}{l}\text { Programa Nacional Beca } \\
18\end{array}$} & \multirow[t]{3}{*}{2014} & Geoffrey Cannock (Coordinador) \\
\hline & & Raúl Andrade Ciudad \\
\hline & & Paul Neira del Ben \\
\hline \multirow[t]{3}{*}{ Materiales educativos } & \multirow[t]{3}{*}{2009} & Martín Benavides Abanto (coordinador) \\
\hline & & Juan José Díaz Noziglia \\
\hline & & Verónica Villarán Bedoya \\
\hline \multirow{3}{*}{$\begin{array}{l}\text { Acompañamiento } \\
\text { pedagógico }\end{array}$} & \multirow[t]{3}{*}{2013} & Luis Felipe Soltau Salcedo (coordinador) \\
\hline & & $\begin{array}{l}\text { José Santos Rodríguez Gonzáles } \\
\text { (Especialista Sectorial) }\end{array}$ \\
\hline & & $\begin{array}{c}\text { María Pilar Sanz Gutiérrez (Especialista en } \\
\text { Evaluación) }\end{array}$ \\
\hline \multirow{3}{*}{$\begin{array}{l}\text { Programa Nacional de } \\
\text { Mantenimiento de locales } \\
\text { escolares }\end{array}$} & \multirow[t]{3}{*}{2009} & $\begin{array}{l}\text { Mayen Lucrecia Ugarte Vasquez Solis } \\
\text { (Coordinadora) }\end{array}$ \\
\hline & & Ricardo David Cuenca Pareja \\
\hline & & María del Carmen Piazza Reyna \\
\hline $\begin{array}{l}\text { Programa Nacional de } \\
\text { Infraestructura } \\
\text { educativa }\end{array}$ & 2012 & Teodoro Sanz Gutiérrez \\
\hline
\end{tabular}

Cuadro 2. Perfil de los responsables de la elaboración de las Evaluaciones de Desempeño y Ejecución Presupuestal en el sector Educación. Elaboración propia.

Con respecto al perfil de quienes elaboraron las EDEP, cabe precisar que la mayoría de las evaluaciones analizadas fueron elaboradas por equipos de consultores conformados por tres miembros. Esto solo varía en la EDEP del Programa Nacional de Infraestructura Educativa, elaborada por un solo consultor. De las cuatro EDEP elaboradas por equipos, tres equipos tuvieron 
conformación mixta, y solo un equipo estuvo conformado exclusivamente por hombres. De los tres equipos mixtos, dos estuvieron compuestos principalmente por hombres, y solo uno contó con mayoría femenina. De manera general, los consultores fueron en mayoría hombres.

En cuanto a la formación profesional en pregrado, sobre el total de consultores la profesión más frecuente es Economía (6), Psicología (3), Sociología (2), Derecho (1) y Educación (1). Si separamos por género, la formación de las consultoras fue la siguiente: Psicología (2), Derecho (1) y Sociología (1); mientras que la formación de los consultores fue la siguiente: Economía (6), Educación (1), Psicología (1), Sociología (1). En todas la EDEP analizadas por lo menos un consultor fue economista, con excepción de la EDEP del Programa Nacional de Mantenimiento de Locales Escolares. Asimismo, la única EDEP donde participó un educador como evaluador fue en la del Programa Nacional Beca 18. Del total de trece consultores, once contaban con alguna maestría (4) o doctorado (6).

Finalmente, ningún consultor o consultora habías postulado a algún cargo de representación política; con excepción de un caso, que postuló el 2016 al Congreso, en la circunscripción Lima, un tiempo después de realizar el informe de la EDEP.

\section{¿Qué metodología emplearon?}

En las EDEP analizadas la metodología empleada fue principalmente cualitativa: se llevaron a cabo entrevistas a diversos actores de Lima y de otros departamentos del país. Adicionalmente, se incorporaron análisis de literatura académica, documentos normativos, informes y bases de datos. Cabe precisar que solo en una EDEP se realizó una correlación entre variables. A continuación, se describe de manera sintética lo concerniente a la metodología empleada en las cinco EDEP según la información disponible en sus respectivos informes.

Para la EDEP del Programa Beca 18 se entrevistaron a diversos actores involucrados en la intervención: Becarios, Instituciones de Educación Superior (Personal administrativo, personal docente, tutores, etc.), personal de UER1/UEL2, miembros de los CVD3 y CVR4, potenciales empleadores. El trabajo de campo se desarrolló en 3 regiones del país: La Libertad (36 entrevistados), Junín (38 entrevistados) y Lima (34 entrevistados). Para seleccionar los referidos lugares se establecieron cuatro criterios: 1) ratio de postulantes sobre el número de becas disponibles, 2) número de becarios por especialista de la UER, 3) número de becarios a graduarse en diciembre $2014,4)$ número de becarios por región.

Por su parte, en la EDEP de Acompañamiento Pedagógico, se entrevistaron a los siguientes actores: trabajadores de las DRE, trabajadores de las UGEL, trabajadores del Gobierno Regional, Formadores, Acompañantes y Docentes acompañados. En el informe se señala que el trabajo de campo se desarrolló en tres lugares, sin embargo solo se proporciona información de dos: San Martín (21 entrevistados) y Tacna (35 entrevistados). Aunque no se menciona explícitamente los criterios de selección de los casos de trabajo de campo, las dos regiones referidas se encuentran dentro del grupo de regiones que demostraron una mejora en logros de aprendizaje de los estudiantes entre el 2009 y

1 Unidades de enlace Regional.

2 Unidades de enlace Local.

3 Comité de Validación Descentralizado

4 Comité de Validación Regional 
2001 (las otras regiones son Ica, Moquegua y Callao), y que se les permitió implementar una focalización alternativa.

Con respecto a la EDEP de Mantenimiento de Locales Escolares, se realizaron entrevistas a los siguientes actores: miembros de Apafa, funcionarios de las UGEL, Directores de Instituciones educativas, autoridades Municipales y a funcionarios del Minedu. A pesar de realizar trabajo de campo en varios departamentos la concentración de los entrevistados fue marcadamente en Lima: Ministerio de educación (7 entrevistados), Lima (18 entrevistados), Arequipa (3 entrevistados) y Piura (1 entrevistado). Cabe resaltar que es la única EDEP donde se realizó una correlación, mostrando que en el plano regional hay una correlación inversa entre los niveles de desarrollo y los montos de subvención del Programa.

En cuanto a la EDEP del Programa Nacional de Infraestructura Educativa se desarrollaron entrevistas a representantes del MINEDU y OINFE en Lima; así como de la DRE, UGEL, GORE, gobiernos locales, y de instituciones educativas de las regiones de Cajamarca, Ica y Lima provincias. En total se entrevistó a aproximadamente 60 personas y se visitaron 20 instituciones educativas. Finalmente, en el informe de la EDEP sobre Materiales Educativos, en lo metodológico solo se señala que se llevaron a cabo entrevistas a los siguientes actores: directivos de DRE, UGEL e IE y docentes de aula.

\section{Niveles de cumplimiento de los compromisos en las matrices del sector Educación al 2017}

a) Programa Beca 18

\begin{tabular}{|l|l|l|}
\hline \multicolumn{1}{|c|}{ Compromiso } & \multicolumn{1}{|c|}{$\begin{array}{c}\text { Medio de } \\
\text { Verificación }\end{array}$} & Cumplimiento \\
\hline $\begin{array}{l}\text { 1.- Generar condiciones para facilitar la transición de } \\
\text { los becarios al mercado laboral }\end{array}$ & 1.1 & No cumplido \\
\cline { 2 - 3 } & 1.2 & No cumplido \\
\hline $\begin{array}{l}\text { 2.- Realizar seguimiento de los becarios egresados y su } \\
\text { inserción en el mercado laboral. }\end{array}$ & 2.1 & No cumplido \\
\cline { 2 - 4 } & 2.2 & No cumplido \\
\hline $\begin{array}{l}\text { 3.- Elevar la rigurosidad y exigencia del proceso de } \\
\text { selección del IES y evaluar anualmente la prestación de } \\
\text { sus servicios. }\end{array}$ & 3.1 & No cumplido \\
\hline $\begin{array}{l}\text { 4.- Proveer información detallada sobre postulación, } \\
\text { inscripción y elección de IES y carreras a todos los } \\
\text { potenciales postulantes. }\end{array}$ & 4.1 & Cumplido \\
\cline { 2 - 4 } & 4.2 & Cumplido \\
\cline { 2 - 4 } & 4.3 & No cumplido \\
\hline
\end{tabular}




\begin{tabular}{|l|l|l|}
\hline & 4.4 & Cumplido \\
\hline $\begin{array}{l}\text { 5.-Optimizar mecanismos de verificación de la } \\
\text { condición de elegibilidad de los becarios. }\end{array}$ & 5.1 & Cumplido \\
\cline { 2 - 4 } & 5.2 & Cumplido \\
\hline $\begin{array}{l}\text { 6.- Reformular la metodología de asignación de cuotas } \\
\text { regionales de becas según demanda efectiva. }\end{array}$ & 6.1 & Cumplido \\
\hline $\begin{array}{l}\text { 7.- Mejorar la calidad de los servicios del sistema de } \\
\text { tutoría para promover un buen rendimiento de los } \\
\text { becarios. }\end{array}$ & 7.1 & No cumplido \\
\cline { 2 - 4 } & 7.2 & Cumplido \\
\hline $\begin{array}{l}\text { 8.- Estandarizar contenidos del ciclo de nivelación para } \\
\text { universidades y establecer un sistema de seguimiento } \\
\text { efectivo. }\end{array}$ & 8.1 & No cumplido \\
\cline { 2 - 4 } & 8.2 & No cumplido \\
\hline $\begin{array}{l}\text { 9.- Considerar los diferentes calendarios según IES en } \\
\text { la programación de fechas de entrega de } \\
\text { documentación. }\end{array}$ & 9.1 & Cumplido \\
\hline $\begin{array}{l}\text { 10.- Planificar mejor el presupuesto para el } \\
\text { cumplimiento de metas de las UER. }\end{array}$ & 10.1 & No cumplido \\
\hline
\end{tabular}

Cuadro 3. Niveles de cumplimiento de los compromisos en las matrices del sector Educación al 2017:

Programa Beca 18. Elaboración propia a partir de información en la página web del Ministerio de Economía y Finanzas (2018).

La EDEP del Programa BECA 18 está compuesta por diez compromisos, que cuentan a su vez con dieciocho medios de verificación. Al respecto, lo primero que se señala es que hasta el informe 2017, los únicos compromisos que se encontraron cumplidos en su totalidad son tres: el 5, 6 y 9 (el compromiso 5 está conformado por dos medios de verificación y los demás por uno). Los compromisos 6 y 9 fueron cumplidos desde el primer informe (2015), y el compromiso 5 fue cumplido de manera progresiva. El cumplimiento de estos compromisos apuntan a mejorar: i) la verificación de la elegibilidad de los becarios (compromiso 5), ii) la selección de los becarios (compromiso 6) y iii) el pago de las subvenciones (compromiso 9).

Por su parte, los compromisos que cuentan con algún medio de verificación cumplido son el compromiso 4 y el 7. El compromiso 4 apunta a brindar información sobre las Instituciones Educativas y carreras a todos los potenciales postulantes; mientras que el compromiso 7 apunta a la mejora el sistema de tutorías. En ambos casos queda pendiente el cumplimiento de un medio de verificación para tener el compromiso totalmente cubierto. El compromiso 4 está compuesto a su vez de cuatro medios de verificación, tres de ellos fueron cumplidos en el primer informe (2015) y el compromiso pendiente de cumplimiento está referido a la elaboración de un manual para las 
Unidades de Enlace Regional y promotores. Por el lado del compromiso 7, el compromiso faltante alude a la elaboración de una directiva referida a la selección de tutores y al contenido de los tutorías.

Son cinco los compromisos que no tienen cumplido ningún medio de verificación, los compromisos 1, 2, 3, 8 y 10. Estos apuntan a la inserción laboral de los becarios al mercado laboral (1), seguimiento de los becarios egresados en su inserción laboral (2), la selección de las Instituciones de Educación Superior y evaluación de las prestación de sus servicios, ciclo de nivelación de universidades (8) y cumplimiento de las metas de las Unidades de Enlace Regional (10). Los compromisos 1 y 2 cuentan con dos medios de verificación, el compromiso 3 y 10 cuenta con un medio de verificación, el compromiso 8 con dos medios de verificación.

Con respecto a los medios de verificación, no hay una relación clara entre el número de medios de verificación y su cumplimiento. Existen dos compromisos que tienen un medio de verificación y no fueron cumplidos (3 y 10), como los hay que sí (6 y 9). De los compromisos que cuentan con dos medios de verificación, existen tres que no cumplieron con ningún medio de verificación $(1,2$ y 8$)$ y solo un compromiso (5) que cumplió con todos los medios de verificación; mientras que otro fue cumplido de manera parcial (7). Solo existe un compromiso que tiene más de tres medios de verificación y fue cumplido de manera parcial (4). Según el último informe 2017, el nivel de cumplimiento de los compromisos ha sido regular (44.4\%).

\section{b) Mantenimiento de locales escolares}

\begin{tabular}{|l|l|l|}
\hline Compromiso & $\begin{array}{l}\text { Medio de } \\
\text { Verificación }\end{array}$ & Cumplimiento \\
\hline $\begin{array}{l}\mathrm{N}^{\circ} \text { 1. Generar una propuesta técnica del diseño } \\
\text { del programa de mantenimiento preventivo con } \\
\text { cambios de corto y mediano plazo }\end{array}$ & 1.1 & Cumplido \\
\cline { 2 - 3 } & 1.2 & Cumplido \\
\hline $\begin{array}{l}\mathrm{N}^{\circ} \text { 2. Implementar un plan de difusión y } \\
\text { capacitaciones para los involucrados }\end{array}$ & 2.1 & Cumplido \\
\cline { 2 - 4 } & 2.2 & Cumplido \\
\hline $\begin{array}{l}\mathrm{N}^{\circ} 3.1 \text { Mejorar los criterios de asignación de los } \\
\text { recursos. }\end{array}$ & 3.1 .1 & Cumplido \\
\cline { 2 - 4 } & 3.1 .2 & Cumplido \\
\hline $\begin{array}{l}\mathrm{N}^{\mathrm{o}} 3.2 \text { Mejorar la definición operativa del } \\
\text { mantenimiento a la infraestructura }\end{array}$ & 3.2 & Cumplido \\
\hline $\begin{array}{l}\mathrm{N}^{\mathrm{o}} 3.3 \text { Mejorar el costeo de los recursos a } \\
\text { transferir por local }\end{array}$ & 3.3 & Cumplido \\
\hline
\end{tabular}




\begin{tabular}{|c|c|c|}
\hline \multirow{3}{*}{$\begin{array}{l}\mathrm{N}^{\circ} 4.1 \text { Reforzar el mecanismo actual para } \\
\text { albergar la información de infraestructura y } \\
\text { vincularlo a los sistemas de información vigentes } \\
\text { (CENSO Escolar, SIAGIE, declaración de } \\
\text { gastos, etc.). }\end{array}$} & 4.1 .1 & Cumplido \\
\hline & 4.1 .2 & Cumplido \\
\hline & 4.1 .3 & Cumplido \\
\hline \multirow{2}{*}{$\begin{array}{l}\text { No } 4.2 \text { Desarrollar un sistema único de } \\
\text { información para la gestión de espacios } \\
\text { educativos en los locales escolares. }\end{array}$} & 4.2.1 & Cumplido \\
\hline & 4.2 .2 & Cumplido \\
\hline \multirow{4}{*}{$\begin{array}{l}\mathrm{N}^{\mathrm{o}} \text { 4.3. Contar con información técnica, primaria } \\
\text { y confiable sobre la situación actual de la } \\
\text { infraestructura de todos los locales }\end{array}$} & 4.3 .1 & Cumplido \\
\hline & 4.3 .2 & Cumplido \\
\hline & 4.3 .3 & No cumplido \\
\hline & 4.3 .4 & No cumplido \\
\hline \multirow{2}{*}{$\begin{array}{l}\mathrm{N}^{\circ} 5 . \text { Generar información estadística anual con } \\
\text { representatividad a nivel nacional y regional para } \\
\text { confirmar el desempeño del programa de } \\
\text { mantenimiento. }\end{array}$} & 5.1 & Cumplido \\
\hline & 5.2 & Cumplido \\
\hline \multirow{2}{*}{$\begin{array}{l}\mathrm{N}^{\circ} \text { 6.1. Desarrollar un sistema de seguimiento y } \\
\text { supervisión que registre datos de programación y } \\
\text { ejecución del mantenimiento a nivel del I.E. }\end{array}$} & 6.1 .1 & Cumplido \\
\hline & 6.1 .2 & Cumplido \\
\hline \multirow{2}{*}{$\begin{array}{l}\mathrm{N}^{\circ} \text { 6.2. Supervisar en campo las acciones de } \\
\text { mantenimiento para una muestra de I.E. }\end{array}$} & 6.2 .1 & Cumplido \\
\hline & 6.2 .2 & Cumplido \\
\hline \multirow{3}{*}{$\begin{array}{l}\mathrm{N}^{\circ} \text { 6.3. Reportar los resultados de seguimiento } \\
\text { en fechas claves. }\end{array}$} & 6.3 .1 & Cumplido \\
\hline & 6.3 .2 & No cumplido \\
\hline & 6.3 .3 & No cumplido \\
\hline $\begin{array}{l}N^{\circ} \text { 7.1. Presentar reportes de la rendición de } \\
\text { cuentas, vinculados a datos de local escolar e } \\
\text { institución educativa. }\end{array}$ & 7.1 .1 & Cumplido \\
\hline
\end{tabular}

Cuadro 4. Niveles de cumplimiento de los compromisos en las matrices del sector Educación al 2017: Mantenimiento de locales escolares. Elaboración propia a partir de información en la página web del Ministerio de Economía y Finanzas (2018). 
La Matriz de Compromisos de Mejora del Mantenimiento de Locales Escolares fue firmada el mes de julio del 2012, por el Ministerio de Educación, a través de la Secretaría de Planificación Estratégica (SPE) y la entonces Oficina de Infraestructura Educativa (OINFE); y por el Ministerio de Economía y Finanzas a través de la Dirección General de Presupuesto Público (DGPP). Asimismo, los compromisos abordaron temáticas diversas, las cuales atañen principalmente al diseño, la gestión presupuestal y el monitoreo y evaluación de los locales escolares.

En cuanto a las limitaciones en el diseño, se observó que tomó al menos tres años lograr una articulación final de la propuesta del diseño técnico del programa, pues en un principio solo se enumeraban las acciones de mantenimiento, pero sin contar con lineamientos claros que los enmarquen y ordenen. Así, si bien para los años 2013-2014 se logró establecer responsabilidades según nivel de gobierno y los procedimientos involucrados para el cumplimiento de las acciones, no es sino hasta el 2015 que se logró detallar objetivos, metas e indicadores.

En relación a la gestión presupuestal, se encontró que entre el 2013 y el 2016 hubo dificultad para definir los criterios de priorización de los locales escolares a intervenir. A ello se le se sumó un costeo incompleto de las acciones de mantenimiento que, si bien se diferenciaba según ámbito geográfico y espacio físico del local escolar (aulas, servicios higiénicos, etc.), dejó de lado otros criterios fundamentales como el estado de la infraestructura, el área geográfica, el tipo de riesgo y si era zona de frontera. Asimismo, los sistemas de información no permitían contar con toda la información desagregada a nivel de local escolar y las acciones de mantenimiento realizadas en cada uno de ellos. Las dificultades para definir dichos criterios de costeo, así como la sistematización ordenada de la información sobre los locales escolares, repercutían directamente en la formulación de las metas físicas y financieras que hacen parte de los programas presupuestales.

Por su parte, es en el seguimiento y evaluación donde se produjo la mayor cantidad de dificultades para implementar las recomendaciones, principalmente relacionadas a la mejora del recojo de información, su sistematización y su traslado oportuno a los actores clave en la toma de decisiones. En ese marco, durante al menos tres años se insistió en la relevancia del Censo de Infraestructura como base para la toma de decisiones, el cual se realizó en el 2013, pero sus resultados no fueron de libre acceso sino hasta el 2016. Los avances sobre el tema fueron reportados solo parcialmente por el MINEDU cada año, lo que afectó directamente la oportunidad de la información, pues al 2016 los datos del Censo ya se encontraban parcialmente "desfasados", considerando que las acciones de mantenimiento continuaban produciéndose. De la misma manera, el establecimiento de mecanismos para la "devolución" de resultados de las acciones de supervisión a las instituciones educativas también tomó al menos tres años, pues si bien se contaba con protocolos y guías de observación, toda la información recabada en el campo no era compartida de forma oportuna. Esta dificultad ha sido parcialmente resuelta con el establecimiento de un solo sistema de registro de las acciones de mantenimiento denominado Wasichachay.

Transversalmente, el Ministerio de Economía y Finanzas observó que en general se producían incongruencias entre las fechas establecidas por las directivas y el inicio del año escolar. Este desfase es crítico, porque el inicio del año escolar es el hito fundamental de la provisión del servicio 
educativo, y al no alinear los procesos de mantenimiento con este, se corre el riesgo de no lograr que las instituciones educativas estén listas para recibir a estudiantes y docentes. Además, los retrasos en el establecimiento de estas fechas afectan a los procesos subsiguientes durante el año.

Al 2017, el nivel de cumplimiento es alto (85,2\%).

\section{c) Materiales educativos}

\begin{tabular}{|c|c|c|}
\hline Compromiso & $\begin{array}{l}\text { Medio de } \\
\text { Verificación }\end{array}$ & Cumplimiento \\
\hline \multirow{2}{*}{$\begin{array}{l}\text { 1.- Elaborar documento oficial "Lineamientos de } \\
\text { política de materiales y recursos educativos } 2013 \text { - } \\
2016 \text { ", en el que se explicitan los lineamientos de } \\
\text { la política de materiales y recursos educativos del } \\
\text { MINEDU para ese periodo, en el marco de los } \\
\text { programas presupuestales con Enfoque de } \\
\text { Resultados de Logros de Aprendizaje. }\end{array}$} & 1.1 & Cumplido \\
\hline & 1.2 & No Cumplido \\
\hline \multirow[b]{2}{*}{$\begin{array}{l}\text { 2.- Definir un flujograma de todo el proceso de } \\
\text { planificación, producción, distribución, desarrollo } \\
\text { de capacidades, monitoreo, uso y evaluación del } \\
\text { kit básico de materiales donde se detallen las } \\
\text { principales actividades y sus respectivos plazos } \\
\text { para que los materiales lleguen a tiempo y los } \\
\text { docentes puedan utilizarlos en la planificación de } \\
\text { sus clases y en el aula. Especificar en este flujo las } \\
\text { variantes que se requieran en la ruta para atender a } \\
\text { la población bilingüe y/o con necesidades } \\
\text { educativas especiales, así como la atención al } \\
\text { enfoque de género. }\end{array}$} & 2.1 & No Cumplido \\
\hline & 2.2 & No Cumplido \\
\hline \multirow[b]{2}{*}{$\begin{array}{l}\text { 3.- Establecer (en coordinación con la OGA) un } \\
\text { plan de adquisiciones multianual de materiales } \\
\text { educativos (2012-2016) donde se detallen todas } \\
\text { las licitaciones de adquisiciones y distribución del } \\
\text { kit básico de materiales por nivel de la EBR y } \\
\text { características de los estudiantes. Además, se debe } \\
\text { consignar un cronograma de ejecución de cada } \\
\text { licitación desde la entrega de las especificaciones } \\
\text { técnicas a la OGA hasta la ejecución de los } \\
\text { procesos, de acuerdo al flujograma establecido. El } \\
\text { cronograma debe tener en cuenta que los } \\
\text { materiales deben estar disponibles en las IE al } \\
\text { inicio del año escolar. }\end{array}$} & 3.1 & No Cumplido \\
\hline & 3.2 & No Cumplido \\
\hline 4.- Revisar y adecuar, conjuntamente con el MEF, & 4.1 & Cumplido \\
\hline
\end{tabular}




\begin{tabular}{|c|c|c|}
\hline \multirow{2}{*}{$\begin{array}{l}\text { la programación de las meta físicas y financieras, } \\
\text { y hacerlas consistentes con el Marco Lógico de los } \\
\text { programas presupuestales de Logros de } \\
\text { Aprendizaje y los indicadores propuestos para su } \\
\text { seguimiento. }\end{array}$} & 4.2 & Cumplido \\
\hline & 4.3 & Cumplido \\
\hline $\begin{array}{l}\text { 5.- Diseñar el proceso operativo de la gestión } \\
\text { administrativa optimizada, los cambios } \\
\text { normativos requeridos y su correspondiente } \\
\text { presupuestación. }\end{array}$ & 5.1 & Cumplido \\
\hline $\begin{array}{l}\text { 6.- (i) Diseñar e implementar un sistema de } \\
\text { monitoreo y evaluación, en base al flujograma } \\
\text { establecido para el ciclo de materiales, que cumpla } \\
\text { con los siguientes objetivos: i) dar cuenta de la } \\
\text { oportunidad, adecuación y suficiencia de la } \\
\text { distribución en cada año; ii) evaluar la concreción } \\
\text { del uso de los materiales en la planificación y } \\
\text { ejecución de las sesiones de aprendizaje de los } \\
\text { docentes, y iii) que cuente con un soporte } \\
\text { tecnológico que agilice la gestión de la } \\
\text { información en las distintas instancias } \\
\text { involucradas. (ii) En el marco del sistema de } \\
\text { monitoreo, diseñar un conjunto de indicadores que } \\
\text { den cuenta de los siguientes aspectos: suficiencia } \\
\text { (que los materiales lleguen a todos los alumnos), } \\
\text { adecuación (en buen estado físico), } \\
\text { contextualización, oportunidad de entrega (antes } \\
\text { del inicio de clases), capacitación en uso a los } \\
\text { docentes, uso de los materiales educativos en el } \\
\text { aula, y resultados esperados. }\end{array}$ & 6.1 & Cumplido \\
\hline \multirow{2}{*}{$\begin{array}{l}\text { 7.- En el marco del sistema de monitoreo, diseñar } \\
\text { un conjunto de indicadores que den cuenta de los } \\
\text { siguientes aspectos: suficiencia (que los materiales } \\
\text { lleguen a todos los alumnos), adecuación (en buen } \\
\text { estado físico), contextualización, oportunidad de } \\
\text { entrega (antes del inicio de clases), capacitación } \\
\text { en uso a los docentes, uso de los materiales } \\
\text { educativos en el aula y resultados esperados. }\end{array}$} & 7.1 & Cumplido \\
\hline & 7.2 & Cumplido \\
\hline
\end{tabular}

Cuadro 5. Niveles de cumplimiento de los compromisos en las matrices del sector Educación al 2017: Materiales educativos. Elaboración propia a partir de información en la página web del Ministerio de Economía y Finanzas (2018).

La Matriz de Compromisos de Mejora de actividades vinculadas a los materiales educativos fue firmada el mes de enero de 2012, por el Ministerio de Educación, a través de la Secretaría de Planificación Estratégica (SPE), la Dirección de Educación Básica Regular (DIGEBR) y la Dirección General de Educación Intercultural Bilingüe y Rural (DIGEIBR), y por el Ministerio de Economía y 
Finanzas, a través de la Dirección General de Presupuesto Público (DGPP). Asimismo, los compromisos abordan temáticas diversas, y atañen principalmente al diseño, la implementación y el monitoreo y evaluación de los materiales educativos.

En primer lugar, como puede verse, la mayoría de los compromisos no cumplidos son aquellos que implican un componente de coordinación entre los actores involucrados. En efecto, en relación al diseño, el Ministerio de Economía y Finanzas recomendó la elaboración de lineamientos de política sobre materiales y recursos educativos, así como la definición de un flujograma de todo el proceso de planificación, distribución, monitoreo y evaluación del kit básico de materiales. Lamentablemente, ninguno de ellos ha sido cumplido hasta el 2017, debido a que no se ha logrado establecer el kit mínimo, el flujograma y las fechas de cada proceso, los roles específicos de los GR y GL5, así como los indicadores e instrumentos para medir el avance físico de la distribución y el logro. De la misma manera, tampoco se logró establecer plazos para la llegada de materiales, así como completar variantes en las rutas de distribución para atender a la población bilingüe y con necesidad especiales, e incorporando un enfoque de género. Como resultado, hasta el 2017 no se lograron aprobar los lineamientos, por lo que se deben actualizarse todos los insumos requeridos a nivel interno del MINEDU.

En segundo lugar, a nivel de la implementación, el Ministerio de Economía y Finanzas realizó sugerencias respecto a la elaboración de un plan multinuanual de adquisiciones, el cual debe ser consistente con lo señalado en el Programa Presupuestal $\mathrm{N}^{\circ} 0090$, y también contar con un proceso operativo de la gestión administrativa optimizada. Por un lado, si bien se lograron mejorar los criterios para la programación de las metas físicas y financieras del PP N ${ }^{\circ} 0090$, así como mejorar ciertos procesos administrativos, no se realizó un diagnóstico de las necesidades de las instituciones educativas según niveles y modalidades; tampoco se identificaron etapas de ni se consignó un cronograma de ejecución. Como resultado, hasta la actualidad aún no existe un Plan Multianual de Adquisiciones, pues los avances hasta el momento son insuficientes.

En tercer lugar, a nivel del monitoreo y la evaluación, se sugirió diseñar e implementar un sistema de monitoreo y evaluación a partir del flujograma establecido para la distribución de materiales, que permitiera dar cuenta de i) la oportunidad, adecuación y suficiencia, ii) la concreción del uso de los materiales en la planificación curricular y iii) la existencia de un soporte tecnológico que agilice la gestión. Asimismo, se propuso diseñar un conjunto de indicadores que permitan evaluar cada uno de los procesos antes mencionados, tomando en consideración además la capacitación a docentes y la contextualización. Sobre estos puntos, si bien desde el 2014 se incluye un módulo para el registro de la distribución de materiales en el Sistema de Información y Gestión para la Mejora de los Aprendizajes (SIGMA), la falta de lineamientos y de un plan de adquisiciones limitan el aprovechamiento de la información registrada por esta herramienta, justamente porque no se encuentran establecidos los objetivos e indicadores cuyo avance debería ser verificado a través de este sistema.

5 No obstante, se debe tener en cuenta que desde el 2015 se cuenta con una Matriz de Gestión Descentralizada, la que al parecer no ha sido reportada por el MINEDU. Sin embargo, en ella se incluye una desagregación de los roles según nivel de gobierno para el proceso de distribución. 
Finalmente, en general se puede concluir que la principal dificultad en el logro de los compromisos para los materiales educativos se encuentra vinculada a la imposibilidad del MINEDU para lograr articular los plazos y fechas para la dotación y distribución del material, debido a que el alineamiento interno de todas las direcciones sigue siendo muy complejo de conseguir. Al 2017, el nivel de cumplimiento fue regular $(61.5 \%)$.

\section{d) Acompañamiento pedagógico}

\begin{tabular}{|c|c|c|}
\hline Compromiso & $\begin{array}{c}\text { Medio de } \\
\text { verificación }\end{array}$ & Cumplimiento \\
\hline \multirow{3}{*}{$\begin{array}{l}\text { 1.- Elaborar paquetes diversificados de } \\
\text { intervención de la estrategia de acompaña- } \\
\text { miento pedagógico (según nivel, ámbito } \\
\text { y/o lengua materna) que responsan a la } \\
\text { diversidad de contextos específicos de la } \\
\text { población. }\end{array}$} & 1.1 & Cumplido \\
\hline & 1.2 & Cumplido \\
\hline & 1.3 & Cumplido \\
\hline $\begin{array}{l}\text { 2.- Elaborar los lineamientos de la } \\
\text { estrategia de acompañamiento pedagógico } \\
\text { que fundamenten los principios y } \\
\text { componentes para la atención específica } \\
\text { de la realidad multi grado, así como los } \\
\text { diferentes modelos y formas de } \\
\text { vinculación entre el MINEDU y los } \\
\text { Gobiernos Regionales }\end{array}$ & 2.1 & Cumplido \\
\hline \multirow{3}{*}{$\begin{array}{l}\text { 3.- Preparar } \\
\text { un ejercicio completo de planificación } \\
\text { estratégica que dé cuenta de la } \\
\text { progresividad de la cobertura del } \\
\text { acompañamiento, considerando diferentes } \\
\text { horizontes temporales y r la } \\
\text { disponibilidad efectiva de recursos } \\
\text { profesionales para su implementación en } \\
\text { cada fase. }\end{array}$} & 3.1 & No cumplido \\
\hline & 3.2 & No cumplido \\
\hline & 3.3 & No cumplido \\
\hline \multirow{2}{*}{$\begin{array}{l}\text { 4.-Desarrollar y difundir el programa } \\
\text { de formación y certificación de } \\
\text { formadores y acompañantes. }\end{array}$} & 4.1 & No cumplido \\
\hline & 4.2 & No cumplido \\
\hline $\begin{array}{l}\text { 5.- Mejorar los procesos de selección, } \\
\text { ingreso, contratación y renovación del } \\
\text { cargo de acompañantes y formadores a } \\
\text { fin de asegurar su disponibilidad en } \\
\text { términos de cantidad y calificación } \\
\text { profesional. }\end{array}$ & 5.1 & Cumplido \\
\hline $\begin{array}{l}\text { 6.- } \text { Estandarizar la información del } \\
\text { trabajo realizado por formadores } \quad \mathrm{y}\end{array}$ & 6.1 & No cumplido \\
\hline
\end{tabular}




\begin{tabular}{|l|c|c|}
\hline $\begin{array}{l}\text { acompañantes pedagógicos y de la } \\
\text { labor de campo, a fin de } \\
\text { evitar duplicidades entre lo que demandan } \\
\text { las instancias intermedias y lo que se }\end{array}$ & 6.2 & No cumplido \\
ingresa en el SIGMA. & & \\
\hline $\begin{array}{l}\text { 7.- Mejorar la información del SIGMA de } \\
\text { manera que recoja variables como: } \\
\text { desagregación por institución educativa }\end{array}$ & & \\
$\begin{array}{l}\text { EIB, procesos cumplido } \\
\text { y de certificación de formadores y } \\
\text { acompañantes. }\end{array}$ & & \\
\hline $\begin{array}{l}\text { 8.- Realizar estudios de las condiciones } \\
\text { actuales de la implementación del }\end{array}$ & & \\
$\begin{array}{l}\text { Acompañamiento Pedagógico, asín } \\
\text { como evaluaciones independientes de sus } \\
\text { efectos en los logros de aprendizaje para } \\
\text { generar evidencias para mejorar }\end{array}$ & 8.1 & Cumplido \\
la estrategia. & 8.2 & Cumplido \\
\hline
\end{tabular}

Cuadro 6. Niveles de cumplimiento de los compromisos en las matrices del sector Educación al 2017: Acompañamiento pedagógico. Elaboración propia a partir de información en la página web del Ministerio de Economía y Finanzas (2018).

La Matriz de Compromisos de Mejora de actividades vinculadas a la intervención de Acompañamiento Pedagógico fue firmada el mes de diciembre del 2014. Asimismo, los compromisos abordan temáticas diversas, y atañen principalmente al diseño, la implementación y el monitoreo y evaluación sobre la intervención.

En primer lugar, respecto al diseño se sugirió diversificar los paquetes de atención, así como los lineamientos para justificar los componentes y modelos, respondiendo principalmente al ámbito y la lengua materna, para hacerlos más adecuados a los contextos. Si bien se logró elaborar los lineamientos y avanzar en los modelos operativos, no se logró concretar una programación presupuestal multianual, lo que impediría un adecuado costeo de los servicios a entregar.

En segundo lugar, respecto a la implementación se propuso mejorar los procesos de contratación y renovación de los acompañantes, así como estandarizar la información del trabajo realizado para evitar incongruencias. En este campo, no se logró diseñar los protocolos y establecer diagnósticos de las necesidades de fortalecimiento de capacidades, así como tampoco se logró estandarizar las características de las visitas en el aula, los microtalleres y los talleres de actualización.

En tercer lugar, a nivel del monitoreo y la evaluación se enfatiza en el mejoramiento del funcionamiento del sistema de gestión de los materiales, así como la realización de estudios sobre la propia intervención que permitan mejorarla. Dichos estudios enfatizaron en i) el acompañamiento multigrado y ii) el acompañamiento bilingüe, dado que son las modalidades en donde más cuellos de botella se han identificado. Para el cumplimiento de este compromiso, se propuso la realización de búsquedas de evidencias, las cuales han llegado a realizarse parcialmente. 
En conclusión, frente al acompañamiento pedagógico se ha detectado sendas dificultades para generar evidencia para la toma de decisiones, lo que se expresa no solo a nivel estratégico y de planificación (para definir los modelos, perfiles, etc.), sino también a nivel operativo, pues no se lograron establecer criterios totalmente claros para la programación presupuestal multianual. Al 2017, el nivel de cumplimiento fue regular (43.8\%).

e) Programa Nacional de Infraestructura Educativa (PRONIED)

\begin{tabular}{|c|c|c|}
\hline Compromiso & $\begin{array}{c}\text { Medio de } \\
\text { Verificación }\end{array}$ & Cumplimiento \\
\hline 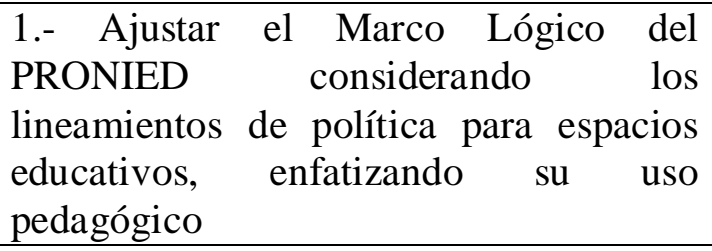 & 1.1 & No cumplido \\
\hline $\begin{array}{l}\text { 2.- Implementar una política de espacios } \\
\text { educativos adecuados a las necesidades } \\
\text { del proceso pedagógico, que guíe el } \\
\text { proceso de planificación y programación } \\
\text { presupuestaria }\end{array}$ & 2.1 & Cumplido \\
\hline $\begin{array}{l}\text { 3.-Implementar el modelo de gestión de } \\
\text { la política de espacios educativos, } \\
\text { identificando procesos operativos, } \\
\text { técnicos y administrativos, y la } \\
\text { interrelación entre los diferentes actores } \\
\text { que puedan contribuir al cumplimiento } \\
\text { de la política }\end{array}$ & 3.1 & No cumplido \\
\hline $\begin{array}{lcc}\text { 4.- Difundir } & \text { los resultados del Censo } \\
\text { Nacional de } & \text { Infraestructura } & \text { Escolar y } \\
\text { establecer } & \text { los } & \text { mecanismos } \\
\text { de actualización del mismo } & \end{array}$ & 4.1 & Cumplido \\
\hline \multirow{2}{*}{$\begin{array}{l}\text { 5. Elaborar el Plan multianual de } \\
\text { inversión y mantenimiento en } \\
\text { infraestructura educativa (a nivel } \\
\text { nacional y de gobiernos sub-nacionales) }\end{array}$} & 5.1 & Cumplido \\
\hline & 5.2 & Cumplido \\
\hline \multirow{2}{*}{$\begin{array}{l}\text { 6. Actualizar y difundir las normas } \\
\text { técnicas y pedagógicas para todos los } \\
\text { niveles y modalidades del servicio } \\
\text { educativo, tanto para infraestructura } \\
\text { como mobiliario y equipos }\end{array}$} & 6.1 & Cumplido \\
\hline & 6.2 & No cumplido \\
\hline \multirow{2}{*}{$\begin{array}{l}\text { 7. Definir una tipología de locales } \\
\text { escolares }\end{array}$} & 7.1 & No cumplido \\
\hline & 7.2 & Cumplido \\
\hline
\end{tabular}




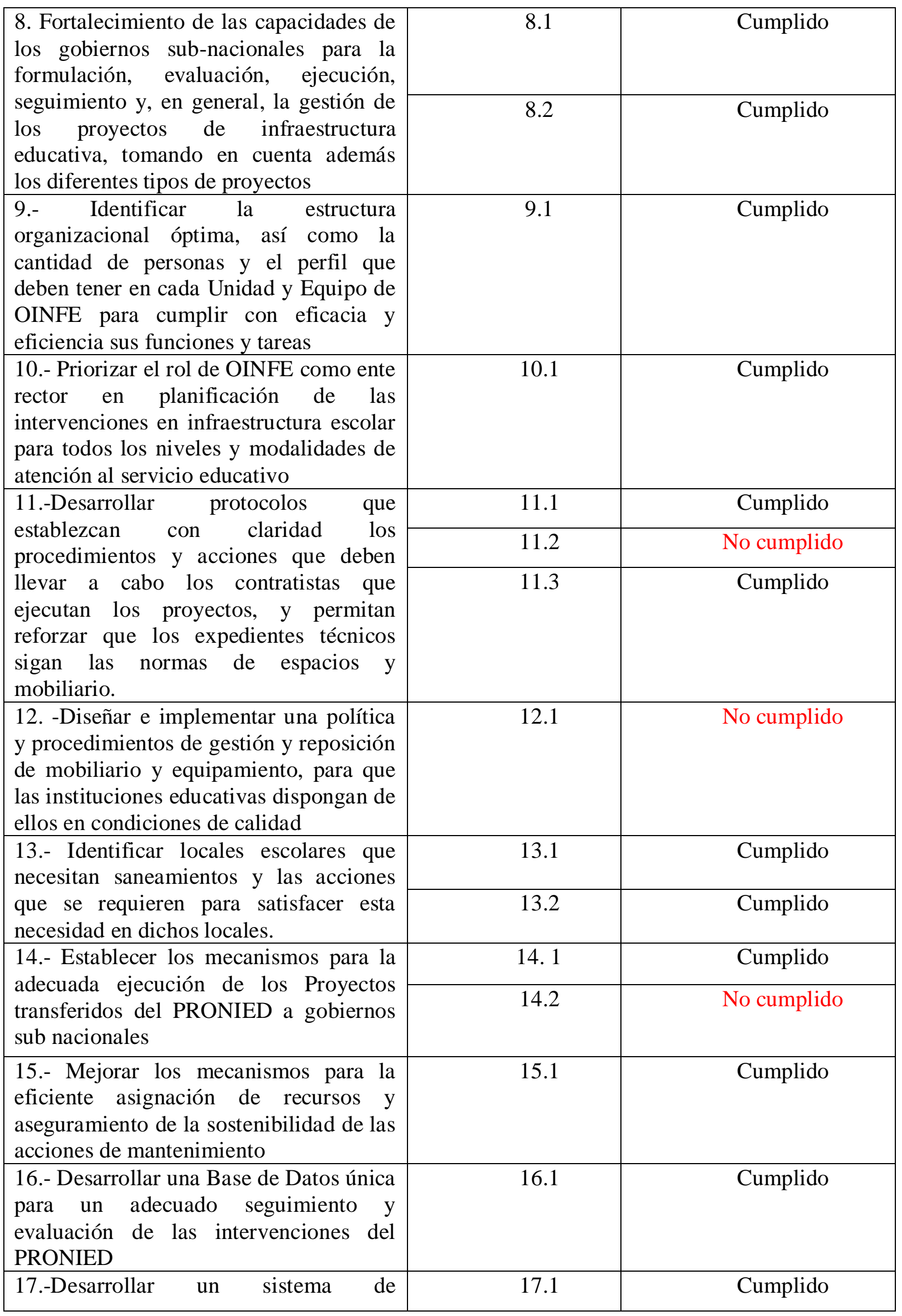




\begin{tabular}{|l|c|c|c|}
\hline seguimiento y evaluación único que & 17.2 & Cumplido \\
registre datos de programación y & & Cumplido \\
ejecución de las inversiones a nivel & & 17.3 & \\
nacional
\end{tabular}

Cuadro 7. Niveles de cumplimiento de los compromisos en las matrices del sector Educación al 2017: PRONIED. Elaboración propia a partir de información en la página web del Ministerio de Economía y Finanzas (2018).

La Matriz de Compromisos de Mejora de actividades vinculadas al Programas Nacional de Infraestructura Educativa-PRONIED fue firmada el mes de julio de 2014. Asimismo, los compromisos abordan temáticas diversas, y atañen principalmente al diseño, la implementación y el monitoreo y evaluación.

En primer lugar, a nivel del diseño, se sugirió la modificación del marco lógico para que este se ajuste a los lineamientos sobre los espacios pedagógicos, así como la difusión de los resultados del Censo (es más, este se publicó recién en el 2016, por lo se encuentra medianamente desactualizado) que pudieran ser utilizados para la toma de decisiones. Asimismo, se sugirió una mayor publicidad y actualización de todos los instrumentos de gestión y la normativa para que puedan ser utilizados de forma oportuna por los actores involucrados. En la práctica, resultó que solamente se logró una actualización parcial de todos los instrumentos de políticas (para la infraestructura, estándares de construcción, mobiliario, etc.), y durante los años en que se ha venido verificando el cumplimiento de la matriz, la actualización de normas ha sido heterogénea. Además, la especificación de criterios para los centros educativos ubicados en zonas rurales tardó bastante tiempo en aclararse.

En segundo lugar, las recomendaciones para la implementación incluyeron el desarrollo de procedimientos más claros para la elaboración de proyectos, así como la identificación de las acciones de infraestructura prioritarias para los locales escolares. También se sugirió el establecimiento de mecanismos de diálogo intergubernamental respecto a la ejecución de proyectos transferidos a los gobiernos sub nacionales. Sin embargo, ninguno de estos compromisos resultó efectivamente cumplido oportunamente, pues solo se reportaron avances pero sin productos específicos.

En tercer lugar, respecto al monitoreo y la evaluación, se sugirió el establecimiento de un sistema de seguimiento y evaluación único para el registro de la programación y ejecución de las inversiones. Frente a esta situación, si bien se logró construir una base de datos única, la propuesta sobre el sistema y su funcionamiento fueron enviados posteriormente. Al 2017, el nivel de cumplimiento fue alto $(74.1 \%)$. 


\section{Conclusiones principales}

En primer lugar, cabe señalar que en líneas generales las evaluaciones han tenido algún grado de cumplimiento -el porcentaje más bajo de cumplimiento es de $44.4 \%$ y el más alto es de $85.2 \%$-. Sin embargo, resulta importante señalar que influye en el nivel de cumplimiento las características de la intervención pública. Por ejemplo, las EDEP con mejor nivel de cumplimiento son sobre todo las intervenciones públicas que se encuentran relacionadas a la infraestructura, mientras que las de menor porcentaje de cumplimiento están directamente relacionadas a las personas (ya sea estudiantes o docentes). Esto se ve reflejado en el ranking de cumplimiento: Mantenimiento de locales escolares $(85,2 \%)$, Programa Nacional de Infraestructura educativa $(74,1 \%)$, Materiales educativos $(61,5 \%)$, Programa Beca 18 (44,4\%) y Acompañamiento Pedagógico (43,8\%).

En segundo término, y en cuanto al perfil de quienes han evaluado los programas, se observa que la mayoría de estas han sido elaboradas principalmente por economistas - que tienen, por lo general, una mayor formación en metodología cuantitativa-. Sin embargo, la metodología empleada en las EDEP ha sido predominante cualitativa realizando trabajo de campo y entrevistas a diversos actores. Si es que esta metodología siguiera siendo la predominante para futuras evaluaciones, sería útil que la composición de los evaluadores fuese un tanto más variada con respecto a las carreras en pregrado, incluyendo perfiles expertos en la temática, por ejemplo educadores.

En tercer lugar, se ha evidenciado que varios compromisos que han presentado mayores dificultades para hacerse efectivos son aquellos que implican la coordinación entre diversas áreas del MINEDU y con otros niveles de gobierno. En efecto, la complejidad para lograr mínimos acuerdos respecto a temas clave como las condiciones de la infraestructura educativa o los Kits de materiales educativos ha impactado directamente en la posibilidad de poder cumplir compromisos que abordan temas de fondo relacionados al establecimiento de lineamientos de política y a los objetivos de la política misma.

En cuarto lugar, dicha complejidad para la toma de decisiones permite esbozar algunos de los límites de las EDEP, así como de los diversos instrumentos de monitoreo y evaluación que se han venido generando. Si bien el enfoque de EBP surge con el objetivo de hacer contrapeso a la arbitrariedad de las decisiones públicas, no puede prescindir enteramente de la arena política ni de las interacciones entre los actores que se mueven alrededor de ellas. Ello no significa negar el valor en sí mismo de la generación de la evidencia, sino por el contrario tener en consideración el cómo la información rigurosa puede hacer parte de la retórica de los actores y sus dinámicas.

Se considera que el impacto de las EDEP aún es limitado, ya que muchas de las recomendaciones atañen a mejorar las visiones y enfoques que subyacen a las políticas educativas, y no a mejorar aspectos operativos concretos. Finalmente, se considera que a pesar de sus limitaciones, las EDEP resultan de utilidad. De hecho, pareciera que no son las evaluaciones EDEP en sí mismas (a diferencia de las evaluaciones de impacto) de mucho alcance para informar modificaciones en una intervención, sino los mecanismos de seguimiento de la matriz de compromiso, fruto de la evaluación. Parece que es la esta matriz y el seguimiento a ella es lo que genera el enforcement para la mejora de las intervenciones. En ese sentido se cuestiona la utilidad de las EDEP como 
mecanismo efectivo de evaluación y mejora del diseño o componente de un programa, y se identifican oportunidades de mejora de la herramienta, como el rediseño metodológico, la simplificación en el reporte de resultados, la potenciación de la matriz y sus mecanismos, entre otros. 


\section{Bibliografía}

Correa, Norma y Mendizabal, Enrique (2011) Vínculos entre conocimiento y política: el rol de la investigación en el debate público en América Latina. Lima: Universidad del Pacífico.

Dargent, E. (2008) Islas de Eficiencia y Reforma del Estado: Ministerios de Economía y Salud 19902008. Proyecto breve abierto. CIES.

Garretón, Manuel y otros (2010) “Ciencias Sociales y Políticas Públicas en Chile: qué, cómo y para qué se investiga en el Estado”. Sociologías. Porto Alegre, año 12, número 24, pp. 76-119. https://doi.org/10.1590/s1517-45222010000200005

Henig, J. (2009). Politicization of evidence: Lessons for an informed democracy. Educational Policy, 23(1), 137-160. https://doi.org/10.1177/0895904808328525

Lee, R., Martin, W., Sontag, H., Taylor, P., Wallerstein, I., Wieviorka, M. (2005) Social Science and Social Policy: from national dilemmas to global opportunities. Reference Paper for the International Forum on the Social Science - Policy Nexus. UNESCO.

Leuz, C. (2018) Evidence-Based Policymaking: Promise, Challenges and Opportunities for Accounting and Financial Markets Research, NBER Working Paper No. 24535.

Milani, C. (2005) Evidence-based policy research: critical review of some international programmes on relationships between social science research and policy-making. Management of Social Transformations Policy Papers, 18, France: UNESCO.

Ministerio de Economía y Finanzas (2009) EDEP sobre Programa de Mantenimiento de Locales Escolares. Lima: MEF. Disponible en:

https://www.mef.gob.pe/contenidos/presu_publ/ppr/eval_indep/2009_mantenimieto_escuelas.p $\underline{\mathrm{df}}$

Consulta: 05 de octubre del 2018.

Ministerio de Economía y Finanzas (2009) EDEP sobre Materiales Educativos. Lima: autor.

Disponible en:

https://www.mef.gob.pe/contenidos/presu_publ/ppr/eval_indep/2009_materiales_educativos.pd $\underline{\text { f. }}$

Consulta: 05 de octubre del 2018.

Ministerio de Economía y Finanzas (2013) EDEP sobre el Programa Nacional de Infraestructura Educativa (PRONIED). Lima: autor. Disponible en: https://www.mef.gob.pe/contenidos/presu_publ/ppr/eval_indep/2009_materiales_educativos.pd f. Consulta: 05 de octubre del 2018.

Ministerio de Economía y Finanzas (2013) EDEP sobre Acompañamiento Pedagógico. Lima: autor. Disponible en: https://www.mef.gob.pe/contenidos/presu_publ/ppr/eval_indep/2013_pedagogico.pdf Consulta: 05 de octubre del 2018. 
Ministerio de Economía y Finanzas (2013) EDEP sobre el Programa Nacional Beca 18 Lima: autor. Disponible en: https://www.mef.gob.pe/contenidos/presu_publ/ppr/eval_indep/2015_beca18_inf_final.pdf Consulta: 05 de octubre del 2018.

Ministerio de Economía y Finanzas (2013) Informe de seguimiento de compromisos de las EDEP al 31 de diciembre del 2012. Lima: autor. Disponible en: https://www.mef.gob.pe/contenidos/presu_publ/ppr/eval_indep/inf_seguimiento_compromisos 2012.pdf. Consulta: 05 de octubre del 2018.

Ministerio de Economía y Finanzas (2014) Informe de seguimiento de compromisos de las EDEP al 31 de diciembre del 2013. Lima: autor. Disponible en: https://www.mef.gob.pe/contenidos/presu_publ/ppr/eval_indep/inf_seguimiento_compromisos 2013.pdf. Consulta: 05 de octubre del 2018.

Ministerio de Economía y Finanzas (2014) Informe de seguimiento de compromisos de las EDEP al 31 de diciembre del 2013. Lima: autor. Disponible en: https://www.mef.gob.pe/contenidos/presu_publ/ppr/eval_indep/Informe_Seguimiento_Compro misos2014.pdf. Consulta: 05 de octubre del 2018.

Ministerio de Economía y Finanzas (2015) Informe de seguimiento de compromisos de las EDEP al 31 de diciembre del 2014. Lima: autor. Disponible en: https://www.mef.gob.pe/contenidos/presu_publ/ppr/eval_indep/Informe_Seguimiento_Compro misos2014.pdf. Consulta: 05 de octubre del 2018.

Ministerio de Economía y Finanzas (2016) Las evaluaciones independientes para la mejora del gasto público. Evaluaciones de Diseño y Ejecución Presupuestal. Lima: autor.

Ministerio de Economía y Finanzas (2016) Informe de seguimiento de compromisos de las EDEP al 31 de diciembre del 2015. Lima: autor. Disponible en: https://www.mef.gob.pe/contenidos/presu_publ/ppr/eval_indep/Informe_Seguimiento_Compro misos2015.pdf. Consulta: 05 de octubre del 2018.

Ministerio de Economía y Finanzas (2017) Informe de seguimiento de compromisos de las EDEP al 31 de diciembre del 2016.2 Lima: autor. Disponible en: https://www.mef.gob.pe/contenidos/presu_publ/ppr/eval_indep/Informe_Seguimiento_Compro misos2016.pdf. Consulta: 05 de octubre del 2018.

Ministerio de Economía y Finanzas (2018) Informe de seguimiento de compromisos de las EDEP al 31 de diciembre del 2017. Lima: autor. Disponible en: https://www.mef.gob.pe/contenidos/presu_publ/ppr/eval_indep/Informe_Seguimiento_Compro misos2017.pdf. Consulta: 05 de octubre del 2018. 
O'Dwyer, L. (2004) A critical review of evidence-based policy making. Australian Housing and Urban Research Institute, Southern Research Centre, AHURI Final Report, 58.

Oliver, K.; Lorenc, T.; Innvær, S. (2014) New directions in evidence-based policy research: $a$ critical analysis of the literature, Health Research Policy and Systems, 12-34. https://doi.org/10.1186/1478-4505-12-34

Pawson, R., Tilley, N. (1996) How (and how not) to design research to inform policymaking, En: C. Samson and N. South (Eds.) The Social Construction of Social Policy: Methodologies, Racism, Citizenship and the Environment. London: MacMillan, 34-52.

Prewitt, K., Schwandt, T., Miron, L. (2012) Using Science as Evidence in Public Policy, Committee on the Use of Social Science Knowledge in Public Policy. Center for Education, Division of Behavioral and Social Sciences and Education, National Research Council. Washington, DC: The National Academies Press.

Veli, E., Becerra, M., García, S., Roca, P., Ruiz, G. (2014) Tecnocracias sociales: El surgimiento de una tecnocracia en el Ministerio de Desarrollo e Inclusión Social. Politai: Revista de Ciencia Política, 5(9), 83-104.

Weiss, C.H. (1979). The many meanings of research utilization. Public Administration Review, 39(5), 426-431. https://doi.org/10.2307/3109916

Zingales, L. (2015) Presidential Address: Does Finance Benefit Society? The Journal of Finance, 70(4), 1327-1363. https://doi.org/10.1111/jofi.12295 